\title{
Source Description of the 1999 Hector Mine, California, Earthquake, Part I: Wavelet Domain Inversion Theory and Resolution Analysis
}

\author{
by Chen Ji, David J. Wald, and Donald V. Helmberger
}

\begin{abstract}
We present a new procedure for the determination of rupture complexity from a joint inversion of static and seismic data. Our fault parameterization involves multiple fault segments, variable local slip, rake angle, rise time, and rupture velocity. To separate the spatial and temporal slip history, we introduce a wavelet transform that proves effective at studying the time and frequency characteristics of the seismic waveforms. Both data and synthetic seismograms are transformed into wavelets, which are then separated into several groups based on their frequency content. For each group, we use error functions to compare the wavelet amplitude variation with time between data and synthetic seismograms. The function can be an L1 + L2 norm or a correlative function based on the amplitude and scale of wavelet functions. The objective function is defined as the weighted sum of these functions. Subsequently, we developed a finite-fault inversion routine in the wavelet domain. A simulated annealing algorithm is used to determine the finite-fault model that minimizes the objective function described in terms of wavelet coefficients. With this approach, we can simultaneously invert for the slip amplitude, slip direction, rise time, and rupture velocity efficiently. Extensive experiments conducted on synthetic data are used to assess the ability to recover rupture slip details. We, also explore slip-model stability for different choices of layered Earth models assuming the geometry encountered in the 1999 Hector Mine, California, earthquake.
\end{abstract}

\section{Introduction}

Detailed mapping of spatial and temporal slip distributions of large earthquakes is one of the principal goals of seismology. After the 1979 Imperial Valley, California, earthquake, an approach called the finite-fault inversion method was developed to study the complexities of larger earthquakes (e.g., Olson and Apsel, 1982; Hartzell and Heaton, 1983). During the past two decades, the methodology has been greatly improved. In the early work of Hartzell and Heaton (1983), the slip velocity was fixed or allowed to vary only slightly during the inversion. Attempts have been made to invert for both slip amplitude and rupture time (e.g., Beroza and Spudich, 1988) and more recently, global inversion methods were introduced, in which slip amplitude, rupture time, rise time are determined simultaneously (e.g., Hartzell et al., 1996). These studies are time-domain inversions; i.e., the fault model is determined by fitting the seismic waveform data. In addition, Olson and Anderson (1988) investigated the use of a linear frequency-domain inversion, which also allows for the simultaneous solution of both slip amplitude and rupture time. Several larger earthquakes have been analyzed using a variety of the above methods (e.g., 1989 Loma Prieta earthquake, Beroza, 1991; Wald et al., 1991; 1992 Landers earthquake, Cohee and Beroza, 1994;
Wald and Heaton, 1994; 1994 Northridge earthquake, Hartzell et al., 1996; Wald et al., 1996), and the complexity and general characteristics of these earthquakes have been used in scores of seismological studies and even to invoke a new theory of earthquake mechanics (e.g., Heaton, 1990).

Although conventional finite-fault inverse procedures work exclusively in either the time domain or the frequency domain, the spatial distribution and character of slip heterogeneity on the fault plane influences not only the frequency content of the outgoing seismic wave, but also when such effects appear on seismograms. To extract more information about slip heterogeneity, it is best to simultaneously consider both the time and frequency characteristics of the waveforms. To this end, we introduce a wavelet transform approach for studying the spatial and temporal slip history of significant earthquakes.

Recently, Graves and Wald (2001) and Wald and Graves (2001) discussed the effects of velocity structure on source resolution. They found that, by using seismic waveform data only, an inaccurate velocity structure could strongly bias the inversion results. Fortunately, they also found that the static displacements have different sensitivity to velocity structure; thus, adding geodetic data to inversion 
enhances the robustness of the inversion. The wavelet transform approach that we will introduce cannot be applied to the static information, however, so we include it in our inversion method separately.

In the present article, we first give a brief review of finite-fault representation theory as suggested by Hartzell et al. (1996), then use it to introduce the wavelet transforms and to construct a useful objective function and perform slip history inversions. Finally, we use the data distributions encountered in the 1994 Northridge and the 1999 Hector Mine, California, earthquakes to test the resolution. In the sequel (Ji et al., 2002), the method developed here was used to study slip history of 1999 Hector Mine earthquake in detail.

\section{Finite-Fault Approach}

The response of a finite fault at a station can be approached by summing the contributions of a regular grid work of subfaults (e.g., Hartzell and Heaton, 1983):

$$
\begin{aligned}
u(t)=\sum_{j=1}^{n} \sum_{k=1}^{n} \mathrm{D}_{j k}\left[\cos \left(\lambda_{j k}\right)\right. & Y_{j k}^{1}\left(V_{j k}, t\right) \\
+ & \left.\sin \left(\lambda_{j k}\right) Y_{j k}^{2}\left(V_{j k}, t\right)\right] \dot{S}_{j k}(t) .
\end{aligned}
$$

Here, $u(t)$ is the displacement at an arbitrary station, $j$ is the $j$ th subfault along strike, and $k$ is the $k$ th subfault down dip. $D_{j k}, \lambda_{j k}$, and $S_{j k}(t)$ are the average dislocation amplitude, rake angle, and rise-time function, respectively. $V_{j k}$ is the average rupture velocity between the hypocenter and subfault $j k$. The terms $Y_{j k}^{1}\left(V_{j k}, t\right)$ and $Y_{j k}^{2}\left(V_{j k}, t\right)$ are the subfault Green's functions for the unit slip in the strike direction and down-dip direction, respectively. Each such function is obtained by summing the responses of point sources uniformly distributed over it. Every point source is delayed appropriately by the time that is equal to the shortest on-fault distance from the hypocenter divided by the average rupture velocity. Thus, all subfault Green's functions separately include the correct effects of the directivity. The number of point sources used depends on the size of subfaults and highest frequency studied.

Following the work of Cotton and Campillo (1995) and Hartzell et al. (1996), we use a modified cosine function to represent the derivative of the rise-time function, $S(t)$ :

$$
\dot{S}(t)=\frac{1-\cos (2 \pi t / r)}{r}, \quad 0<t<r .
$$

Here, the $r$ is the width of the rise-time function. The advantage of this approach is that we need only one parameter to represent the rise-time function. This makes the inversion more stable, but it limits the complexity of the time history of individual subfaults in comparison to the multiple-timewindow approach (e.g., Wald and Heaton, 1994). However, Guatteri and Spudich (2000) have demonstrated that such complexity may be hard to constrain with only lowfrequency strong-motion data.
Equation (1) can also be used for the static response of a finite fault by replacing $Y_{j k}^{i}\left(V_{j k}, t\right)$ with its static Green's function $Y_{j k}^{i}$. With this approach, a full representation of the fault response relies on four parameters: dislocation amplitudes, rake angles, average rupture velocities, and rise-time widths. Thus, we can invert the parameters by matching the synthetic seismograms and static displacements to the observations.

\section{Objective Function and Inversion Method}

Finite-fault inversion involves finding the values of fault parameters that can minimize a misfit or objective function. This function characterizes the differences between observed and synthetic data calculated by using a fault model and the propagation effects produced by an assumed Earth model. Hence, two questions must be answered: what is the definition of the misfit function, and how do we find the minimum? We will address these issues in this section.

For significant earthquakes, the relative low-frequency signals (frequency $f<0.2 \mathrm{~Hz}$ ) usually dominate displacement records. Thus, such information is essential to constrain the general picture of a seismic source, but it is not as sensitive to the detailed characteristics, such as variations of rise time or rupture velocity. On the other hand, a sudden change in slip amplitude or rupture velocity radiates strong high-frequency seismic signals (effects similar to a stopping phase; Aki and Richard, 1980). Hence, studying higherfrequency signals will increase the spatial and temporal resolution.

Because of the large difference in amplitudes, however, it is difficult to simultaneously capture lower- and highfrequency information with time-domain waveform inversions, which emphasize the longer-period and largeramplitude signals. While the fit to lower-frequency and higher-frequency signals are independent in frequencydomain inversions, because Fourier transform decomposes seismograms into sine or cosine functions, it is easy to lose other important information about when such signals arrive.

To handle this problem, we could separate a seismogram into many traces containing different frequencies and invert them separately. This was attempted by Mendoza and Hartzell (1988), who used long-period, short-period, and intermediate-period teleseismic $P$ waves to constrain the slip distribution for the 1986 Palm Springs earthquake. Similarly, Wald et al. (1996) used both displacement and velocity records for the slip history of 1994 Northridge, California, earthquake. Here, we introduce a new data processing technique, the wavelet transform, which appears to be ideal for handling the above time-frequency problems, and is also ideal for inversion application.

\section{Wavelet Transform}

The wavelet transform was developed and applied in the last two decades (Mallat, 1998). In contrast to the Fourier 
transform, it decomposes the time series into a sum of wavelets, which are functions $\psi(t)$ satisfying the rule

$$
\int_{-\infty}^{+\infty} \psi(t) \mathrm{d} t=0 .
$$

The wavelet function dilated with a scale parameter $s$ and translated by position $u$ is referred to as a wavelet atom:

$$
\psi_{s, u}(t)=\frac{1}{\sqrt{s}} \psi\left(\frac{t-u}{s}\right) .
$$

A wavelet transform coefficient of seismogram $f(t)$ at the wavelet scale $s$ and position $u$ is defined by

$$
\alpha_{s, u}=\int_{-\infty}^{+\infty} f(t) \psi_{s, u}(t) \mathrm{d} t .
$$

With such continuous wavelet transforms, we can detect signal variations in $2 \mathrm{D}$ time-frequency space. It is not suitable for an inversion procedure, however, because $s$ and $u$ are continuous and do not form an orthonormal base by uniformly sampling (Mallat, 1998). Hence, we will either miss some useful information or get abundant linearly dependent constraints. This problem can be solved by using an orthonormal discrete wavelet transform (ODWT). In fact, one ODWT, called the Meyer-Yamada wavelet (MYW), has been introduced to process seismic data by Yomogida (1994). The analytic representation of MYW $\psi$ is complex, as discussed by Yomogida (1994). Here, we plot its shapes in the time and frequency domains in Figure 1 to illustrate its basic characteristics. Note that in the frequency domain, the real parts of $\psi(\omega)$ are symmetric and the imaginary parts are antisymmetric, thus $\psi(t)$ is a pure real function in the time domain, which is a particularly useful property in handling time series.

For a discrete time series $y_{i}(i=0,1,2, \ldots, N-1$, and $N=2^{n}$, where $n$ is an integer) with the length $T(T=$ $N \Delta t$, where $\Delta t$ is the sample interval), the discrete MYW atoms can be represented as

$$
\begin{aligned}
\psi_{j, k}(i) & =\sqrt{\frac{1}{2^{n-j} \Delta t} \psi\left(\frac{i}{2^{n-j} \Delta t}-k\right),} \\
j & =0,1, \ldots, n-1 \text { and } k=0,1, \ldots, 2^{j-1} .
\end{aligned}
$$

Here, $j$ is the discrete form of $s, s=2^{n-j} \Delta t$, and $k$ is the discrete form of $u, u=k \cdot 2^{n-j} \Delta t$. The discrete wavelet transform is expressed by

$$
y_{i}=\sum_{j=0}^{n-1} \sum_{k=0}^{2^{j}-1} \alpha_{j, k} \psi_{j, k}(i)
$$
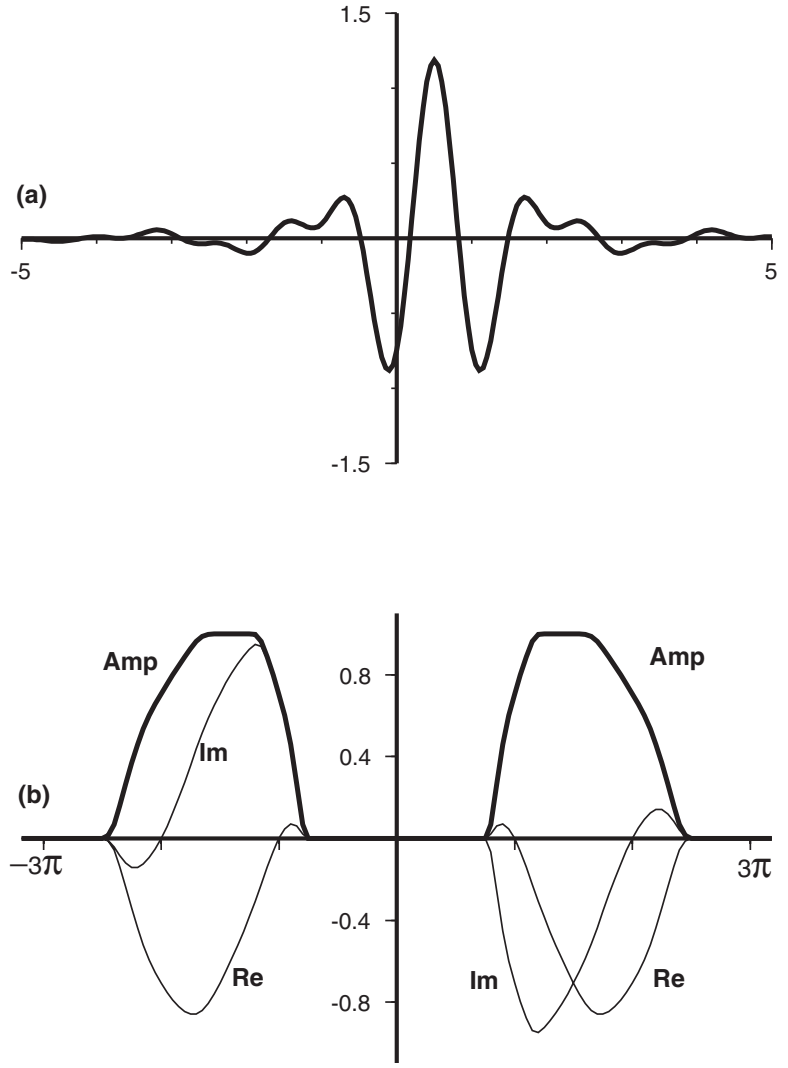

Figure 1. Analyzing Meyer-Yamada wavelet. (a) Waveform in the time domain. (b) Amplitude (thick line), real (thin line) and imaginary (dashed line) parts in the frequency domain (modified from Yomogida, 1994).

where the coefficients $\alpha_{j, k}$ are calculated with the fast algorithm suggested by Yamada and Ohkitani (1991).

Since the wavelet function $\psi$ will be nonzero only within $[2 \pi / 3,8 \pi / 3]$ (Fig. 1), the wavelet atom $\psi_{j k}(\mathrm{t})$ is bandlimited in range $\left[2^{j} / 3 T, 2^{j+2} / 3 T\right]$ for each $j$; Furthermore, in contrast with the sine and cosine harmonic functions, which have the same amplitudes universally, the wavelet function is compact. The amplitude decay in Figure 1a shows a factor of 10 reduction over a small window $-1<s<2$. Hence, a coefficient $\alpha_{j, k}$ measures the variation of $s_{i}$ in the neighborhood of $i=k \times 2^{n-j}$, whose size is proportional to $s$. However, because of the condition shown in equation (3), the wavelet transform cannot resolve the static component of the signal. This is consistent with the fact that the total number of coefficients is $N-1$, even though the number of original data is $N$ (Yomogida, 1994).

The MYM is an orthonormal base (Yomogida, 1994); i.e., suppose we have another time series $u_{i},(i=0,1, \ldots$, $N-1)$ with corresponding coefficients $\beta_{j, k}$. Then $u_{i}$ is a good match to $y_{i}$ if and only if $\alpha_{j, k}=\beta_{j, k}$, for $j=0,1, \ldots$, $n-1 ; k=0,1, \ldots, 2^{j}-1$. With this characteristic, we can construct an objective function in the MYM domain, as we did in the time and frequency domain. 


\section{Test Example}

We use a simple test to show the advantage of our timefrequency analysis. A fault model that is simplified from the finite-fault inversion modeling of 1994 Northridge earthquake (Fig. 2; Wald and Heaton, 1996) is used to generate the synthetic data. Note that we assign slip to occur only on two rectangle asperities, A and $\mathrm{B}$. Asperity A is deep; it has a uniform rise time of $0.5 \mathrm{sec}$ and slip of $1 \mathrm{~m}$. Asperity B is shallower, has $2.5 \mathrm{sec}$ rise time and $2 \mathrm{~m}$ slip.

We generate the synthetic $P$ waves of an arbitrary teleseismic station, whose distance and azimuth are $70^{\circ}$ and $236^{\circ}$ respectively. We choose a layered velocity model suggested by Wald et al., (1996). The responses to asperity A, B, and the whole fault $(A+B)$ are shown in (Fig. 2b).

Due to the differences in propagation and rise time, the waveforms generated from the two patches are quite different. The response generated from subevent A has more highfrequency energy and arrives earlier; that from the subevent $\mathrm{B}$ has more lower-frequency signal and arrives later. For the purpose of inversion, we attempt to find the crucial information from $\mathrm{A}+\mathrm{B}$ that can separate the effects of the two asperities. Note that it is the high-frequency information that highlights the differences in rise time, and it is the time information that can localize the position of source.

In Figures $2 \mathrm{c}$ and $2 \mathrm{~d}$, we use the MYW transform to convert the data A and B into traces with the same scales and compare them with corresponding transform results of data $\mathrm{A}+\mathrm{B}$. Note that it becomes easier to separate the effects in the time-frequency domain (Figs. 2c and 2d) than in the time domain only (Fig. 2b). First, the energy from the short rise-time subevent dominates the higher-frequency channels and that from the long rise-time subevent is more apparent in the lower-frequency channels. Second, the wavelet transform can provide relatively accurate localization of seismic energy in the high-frequency channels; e.g., in the last three channels $(j=6,7,8)$, the signals have died out before the end of the signal generated by block B. This appears to be the key information needed to constrain rise-time variations.

However, the wavelet transform cannot change the frequency content of a seismic signal. The amplitude difference between high-frequency and lower-frequency signals still exists. Hence, if we just use the L2 norm as the criterion for measuring the difference between wavelet atom coefficients, we would get the same results as we do in the time domain. As a test, we assume the synthetic seismogram of A + B is $o_{i}, i=0,1, \ldots, 511$. The sample interval is $0.1 \mathrm{sec}$ and the $\alpha_{j, k}$ are wavelet coefficients. We define another series, $s_{i}^{m}$, which satisfy

$$
s_{i}^{m}=\left\{\begin{array}{cl}
0 & \text { if } m=0 \\
\sum_{j=0}^{m-1} \sum_{k=0}^{2^{j}-1} \alpha_{j, k} \psi_{j, k}(i) & \text { if } 0<m<9 .
\end{array}\right.
$$

We can measure the least-square error in both time and wavelet domains by the formula

$$
e_{m}^{t}=\frac{\sum_{i=0}^{N-1}\left(o_{i}-s_{i}^{m}\right)^{2}}{\sum_{i=0}^{N-1} o_{i}^{2}}
$$

and

$$
e_{m}^{w}=\frac{\sum_{j=m}^{n-1} \sum_{k=0}^{2^{j}-1} \alpha_{j, k}^{2}}{\sum_{j=0}^{n-1} \sum_{k=0}^{2^{j}-1} \alpha_{j, k}^{2}} .
$$

Here, $e_{m}^{t}$ and $e_{m}^{w}$ are the normalized L2 errors in the time domain and the wavelet domain, respectively. The crosses and circles in Figure 3 show the variation of two error functions with $m$, which essentially overlie. This test, in fact, is a demonstration of the orthonormal characteristic of MYW. This example also indicates that time-domain inversion emphasizes longer-period and larger-amplitude signals. Note that after summing the first five channels $(j>5)$, the error function is reduced to less than $5 \%$. It is noteworthy that we need only 31 wavelet coefficients to uniquely determine the waveforms of the first five channels $(j=0,1,2,3,4)$, which is only about $6 \%$ of the total number of coefficients (511). Nonetheless, if we cannot use information of smaller-scale signals, the constraint of the source is strongly limited. Furthermore, the key information that can separate differences in rise time is contained in the small-scale traces. Fortunately, in the wavelet domain, we can separate the coefficients by scales, and then the contribution of the smallerscale coefficients can be enhanced easily.

Finally, it is noteworthy that using the wavelet transform to divide the time series into traces with the same scale is similar in effect to the multiple-bandpass filter. Hence, the method proposed here is close to the approach used by Mendoza and Hartzell (1988). However, constructing the error function in the wavelet domain is more efficient than in the time domain; suppose we have a seismogram with $N$ samples: if we separate it into $M$ frequency bands, then we need match $N M$ time samples, compared with $N$ coefficients, for the wavelet transform approach.

\section{Objective Function for Waveform: Multiple Criteria}

Suppose series $o_{i}$ and $y_{i}$ are the observed and synthetic seismograms, respectively, and $o_{j, k}$ and $y_{j, k}$ are the corresponding wavelet coefficients. As we have discussed, the large amplitude difference between the lower- and higherfrequency signals still exists; thus, we need to give more weight to the coefficients of small-scale (high-frequency) wavelet atoms. For this purpose, we separate the wavelet 


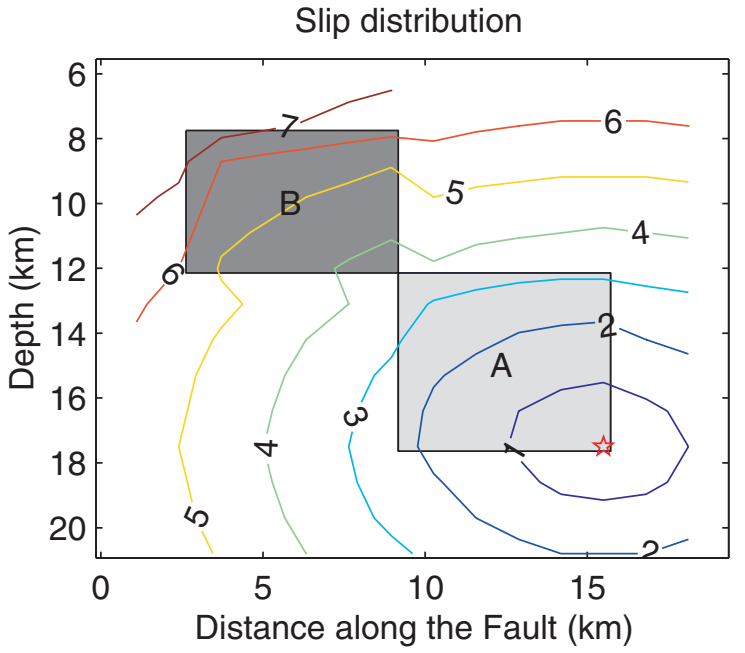

(a)

\section{$\mathrm{A}+\mathrm{B}$ vs. $\mathrm{A}$}

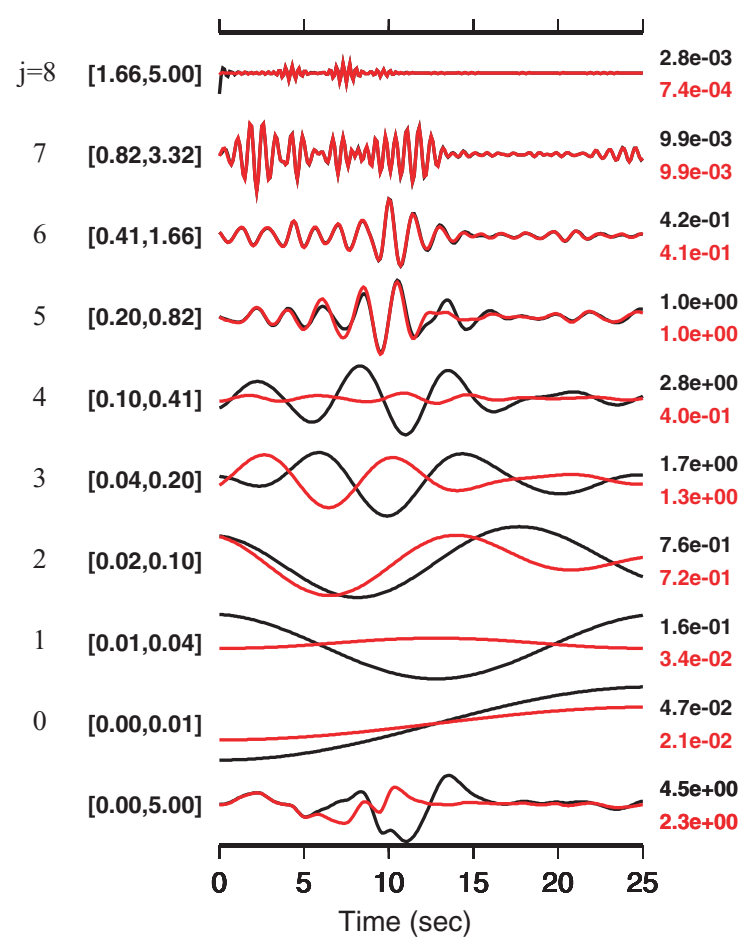

(c)

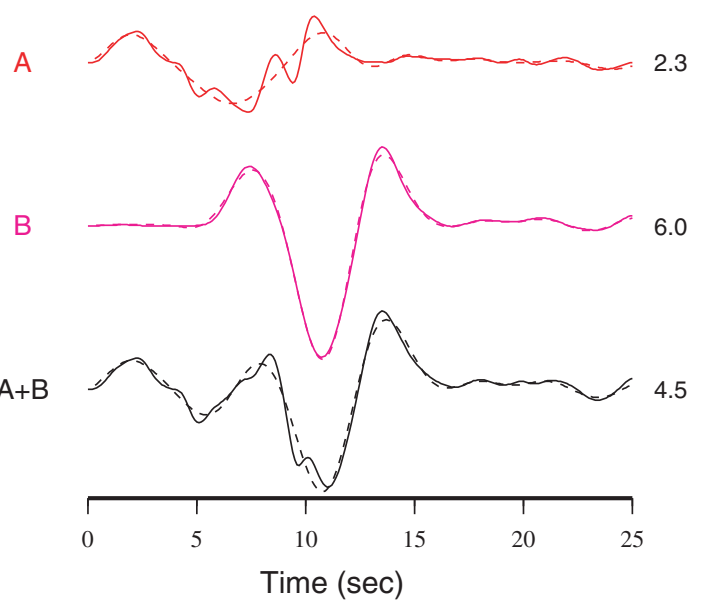

(b)

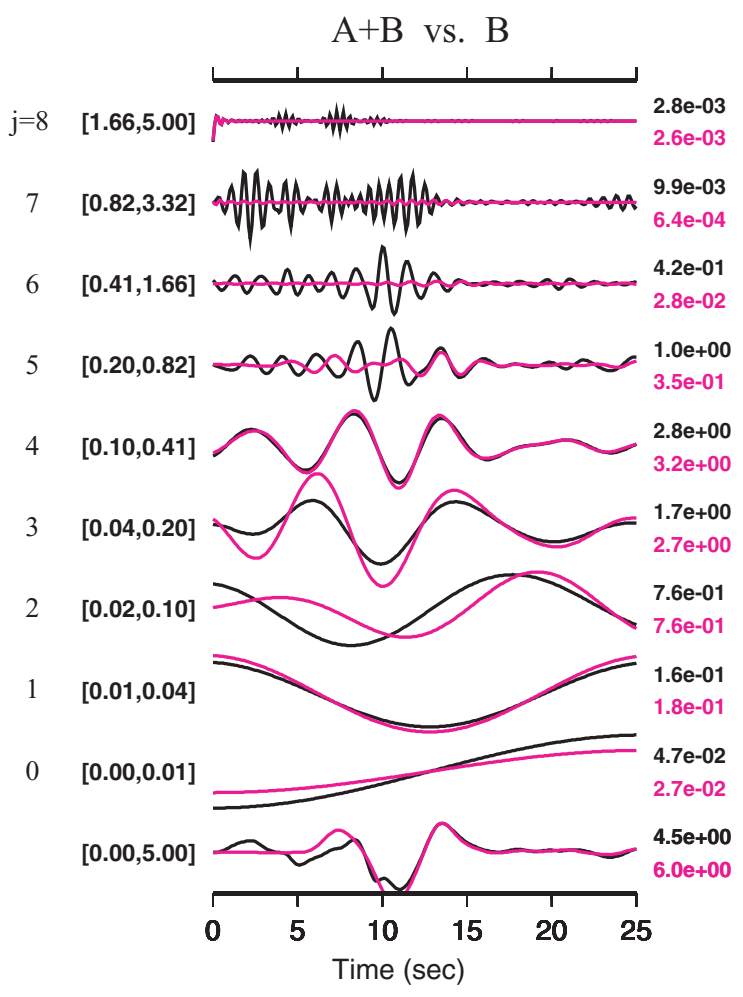

(d)

Figure 2. The advantage of wavelet transform. (a) The dip, strike, and rake angles of the test fault plane are $40^{\circ}, 121^{\circ}$ and $112^{\circ}$, respectively. The slip distribution has been discussed in text. Contours in a 1.0 -sec interval indicate the rupture initiation time. (b) Synthetic vertical velocity records of teleseismic $\mathrm{P}$ wave are generated by the block A, block B, and whole fault $(\mathrm{A}+\mathrm{B})$ as labeled in the left, respectively. The dashed lines shown the contributions in the first 5 wavelet scales $(j=0,1,2,3,4)$. The peak amplitudes in $\mathrm{mm} / \mathrm{sec}$ are indicated in the right. (c) Comparison between data $\mathrm{A}$ and data $\mathrm{A}+\mathrm{B}$ in different wavelet channels. The frequency content of each wavelet channel is shown in the left (low, high frequency bands given), and the peak amplitudes are indicated in the right with the same color as data. (d) Similar comparison between data $\mathrm{B}$ and data $(\mathrm{A}+\mathrm{B})$. 
coefficients into two groups by their scales and use different criteria to measure them.

The L1 or L2 norm is good at comparing absolute amplitudes; their combination takes advantage of both L1 and L2, as suggested by many authors (e.g., Zhao and Helmberger, 1994). We use it to measure the differences in lowerfrequency (long scale) but larger-amplitude coefficients:

$$
\begin{aligned}
e_{l}=\sum_{j=j_{\min }}^{j_{\mathrm{c}}} \mathrm{W}_{\mathrm{j}}\left(\frac{1}{k_{j}} \sum \mid o_{j, k}\right. & -y_{j, k} \mid \\
& \left.+\sqrt{\frac{1}{k_{j}} \sum^{k_{j}}\left(o_{j, k}-y_{j, k}\right)^{2}}\right) .
\end{aligned}
$$

Note that the largest wavelet scale $s_{\max }$ used in an inversion is equal to $T / 2^{j_{\mathrm{min}}}$ and the critical scale $s_{\mathrm{c}}$ can be represented as $s_{\mathrm{c}}=T / 2^{j_{\mathrm{c}}}$, where $T$ is the signal duration. Thus $j_{\min }$ and $j_{\mathrm{c}}$ correspond to the largest- and smallest-scale wavelets measured by these criteria, respectively.

The correlative function suggested by Sen and Stoffa (1991) is focused on signal shape; it is relatively less sensitive to the signal amplitudes, and so is good at handling the high-frequency and small-amplitude information. Thus, we define

$$
e_{\mathrm{h}}=\sum_{j_{\mathrm{c}}+1}^{j_{\max }} W_{j}\left(1.0-\frac{2 \sum^{k_{j}} o_{j, k} y_{j, k}}{\sum o_{j, k}^{2}+\sum_{j}^{k_{j}} y_{j, k}^{2}}\right)
$$

Here, $j_{\max }$ corresponds to the smallest-scale wavelets, $s_{\min }$ $=T / 2^{j_{\max }}$.

As we pointed out earlier, the effect of the wavelet transform is similar to multiple-bandpass filters. By defining the bandpass width of a wavelet function as the frequency region with spectrum amplitudes larger than $\sqrt{2} / 2$, we can derive the simple relation between the bandpass width and scale of a wavelet atom; i.e., $1 / 2 s<f<1 / s$, where $f$ is the frequency. Hence the choices of $s_{\max }$ and $s_{\min }$ should depend on the frequency contents of the seismic signals, and our knowledge of the Earth structure. In our study, $s_{\max }$ is roughly equal to the reliable signal duration; $s_{\min }$ is close to the shortest seismic period that we can model.

The critical scale $s_{\mathrm{c}}$ is chosen based on the amplitude variation with wavelet scales. For example, in the later simulations of 1994 Northridge, California, earthquake, we use $s_{\mathrm{c}}$ as $1.6 \mathrm{sec}$. In the work on 1999 Hector Mine, California, earthquake, however, we use a longer scale, $s_{\mathrm{c}}=3.2 \mathrm{sec}$, because in that event, most strong-motion records were dominated by the lower-frequency signals (Fig. 5 in our companion article, Ji et al., 2002).

Figure 4 shows the flow chart of the objective function. This function is the sum of measurements of the higher frequency channels and lower frequency channels:

$$
\operatorname{err}_{\mathrm{wf}}=e_{1}+e_{\mathrm{h}}
$$

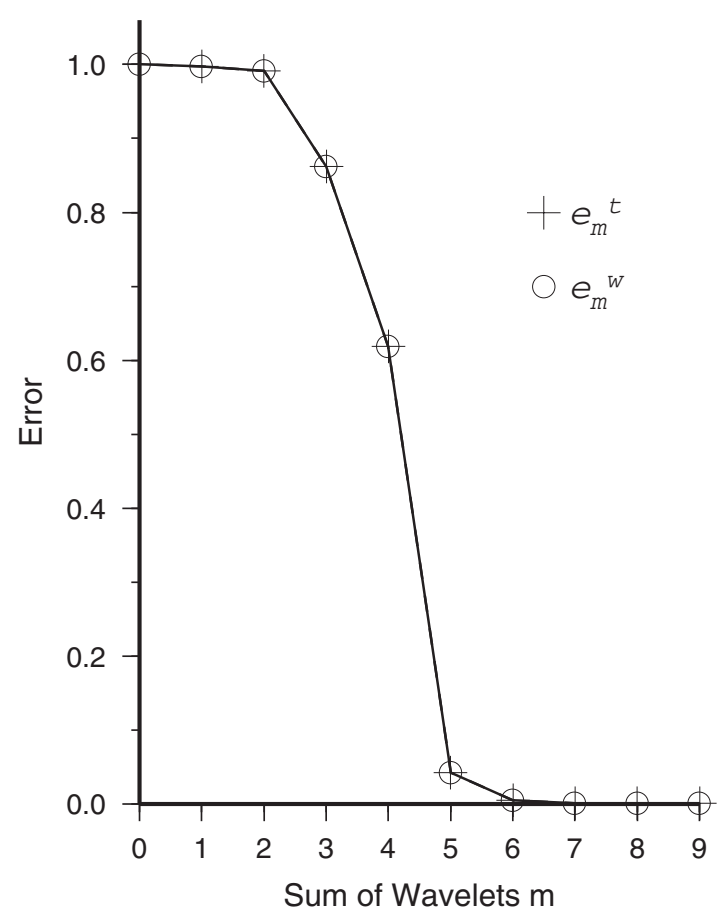

Figure 3. L2 norm variation versus sum of wavelet channels. The crosses show the behavior in the time domain; circles show the behavior in the wavelet domain.

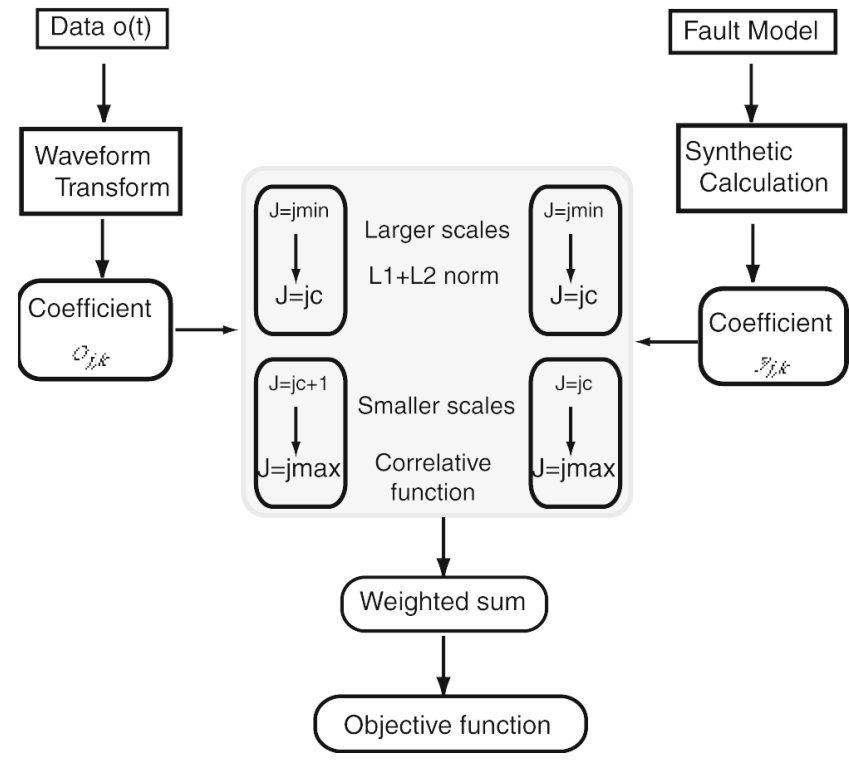

Figure 4. Flow chart of the objective function. Note that the synthetic calculation includes two steps. First, we generate synthetic seismograms in the time domain and then we do a wavelet transform to convert them into the wavelet domain. 
Note that the data need to be transformed only once. However, for each new fault model, the synthetic wavelet coefficients must be recomputed. In our approach, both teleseismic and strong motion synthetic seismograms are generated in the frequency domain, thus we can apply the efficient algorithm suggested by Yamada and Ohkitani (1991) to calculate the wavelet coefficients.

\section{Objective Function for GPS Measurement}

Near-field GPS measurements are very helpful in constraining the slip distribution of earthquakes and thus increase the robustness of inversion (Wald and Graves, 2000). As we have mentioned, however, static displacement data cannot be included into the wavelet transform approach. Thus, we need to treat the static displacement separately.

Sum-squared residuals $\left(\chi^{2}\right)$ have been used as the criteria for measuring the difference between synthetic and observed static displacements (e.g., Hudnut et al., 1996). This can be represented as

$$
\operatorname{err}_{\mathrm{st}}=\sum_{i}^{N}\left(\frac{S_{\mathrm{o}}^{i}-S_{\mathrm{s}}^{i}}{\sigma_{i}}\right)^{2}
$$

where $\mathrm{S}_{\mathrm{o}}^{i}$ and $\mathrm{S}_{\mathrm{s}}^{i}$ are the $i$ th observed and synthetic static displacements, respectively, and $\sigma$ is the observed standard error. However, this criterion has two weaknesses. First, since the static displacements decay rapidly with the distance from fault trace, the formula cannot fairly treat every measurement. For instance, in our study of the 1999 Hector Mine earthquake (Ji et al., 2002), the largest GPS record is $85 \mathrm{~cm}$ and the smallest is only $3 \mathrm{~cm}$. Suppose $\sigma$ is the same for all data and that the criterion $\chi^{2}$ indicates that there is a $1.5-\mathrm{cm}$ misfit in some measurements. Then, if such misfit is related to the largest observation, the model can explain greater than $98 \%$ of the amplitude. Thus, further improvement is better but may be not necessary. If the same amount of misfit is relative to the $3.0-\mathrm{cm}$ observation, however, the model should be further improved. Second, equation (14) only considers the observed error, but our numerical example discussed later demonstrates that two possible layered velocity structures can easily produce $5 \%$ amplitude differences.

Due to the uncertainty in Earth structure, a perfect fit to the largest-amplitude records may not be a physical or desirable solution, but a model is probably acceptable if it can explain greater than $95 \%$ of the amplitude of every GPS observation. Thus, we modified equation (14) by introducing a threshold. If the relative difference between the synthetic static displacement and the data is less than $5 \%$, we let the difference be zero. Then, the new criterion is

$$
S_{\mathrm{o}}-S_{\mathrm{i}}=\left\{\begin{array}{lr}
0 & \text { if }\left|S_{\mathrm{o}}-S_{\mathrm{i}}\right|<0.05 S_{\mathrm{o}} \\
S_{\mathrm{o}}-S_{\mathrm{i}} & \text { otherwise }
\end{array} .\right.
$$

With this precondition expression (15), the result will have an error even when err $_{\text {st }}$ is zero. It is very small, however, and we can prove that the errors satisfy

$$
\frac{\Delta S}{S} \leq 0.05, \quad \Delta \theta<3^{0}
$$

$\Delta S$ and $\Delta \theta$ are the probable misfits in amplitude and direction when the $\chi^{2}=0.0$.

\section{Inversion Method}

When we try to simultaneously invert seismic and GPS observations, the criteria can be represented as

$\operatorname{err}_{\mathrm{wf}}+W_{\mathrm{st}} \cdot \mathrm{err}_{\mathrm{st}}+W_{\mathrm{c}}$ (constraints) $=$ minimum.

Two types of constraints are chosen: one that minimizes the difference between the slip on adjacent subfaults and a second that minimizes the total moment (Hartzell et al., 1996). Using additional constraints increases the stability of inversion, but the resolution is reduced. Two weights $W_{\mathrm{st}}$ and $W_{\mathrm{c}}$ are used to adjust the trade-off between fitting the two different data sets and satisfying the constraints. Their values are obtained on a trial-and-error basis, to ensure that the fits to two data sets are not strongly degraded. In our procedure, all inversions start with random initial fault models with total slips equal to the result of the point source inversions (e.g., centroid moment tensor [CMT] solution). The inversions with individual datasets and no constraints are first per-

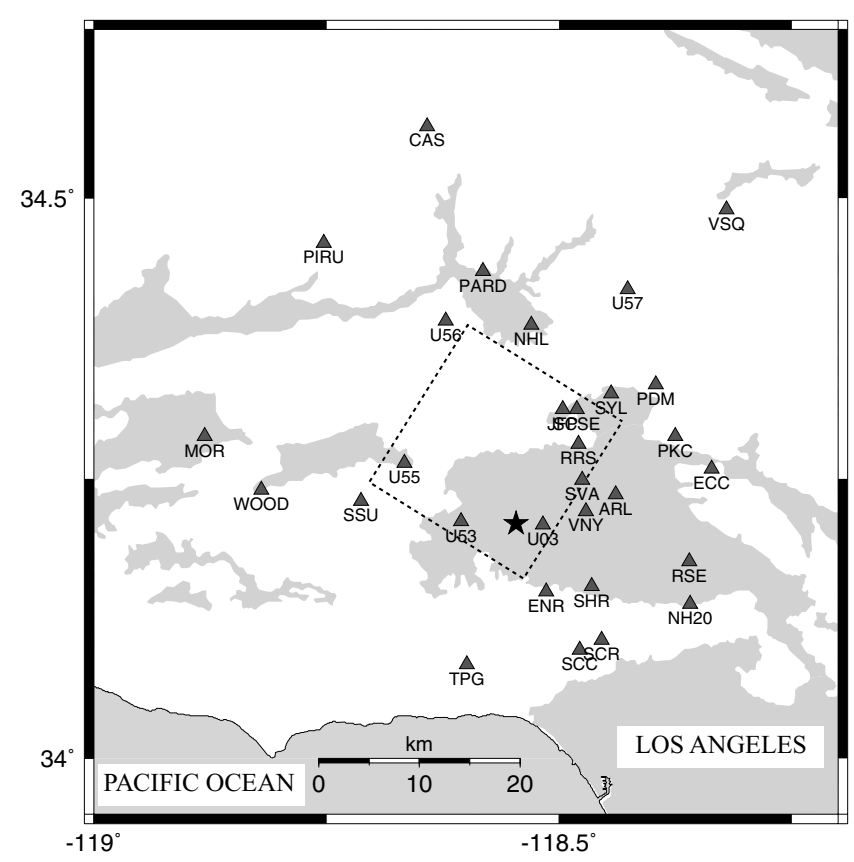

Figure 5. Projection of the 1994 Northridge earthquake fault plane and strong motion stations (triangles). The star shows the location of the epicenter. 
formed to determine the possible maximum improvements, which are then used to normalize the $\operatorname{err}_{\mathrm{wf}}$ and $\mathrm{err}_{\mathrm{st}}$ in the future combined inversion. The smoothing constraints are also normalized with the value of the initial models. After this process, the weights $W_{\text {st }}$ and $W_{\mathrm{c}}$ become dimensionless.

We choose a particular version of the simulated annealing (SA) inverse method, called the heat-bath algorithm (Rothman 1986), to search for the best finite-fault model in the whole model space. Compared to other simulated annealing algorithms, it has two advantages. First, it acts by perturbing the objective parameters one by one. When we perturb the parameters of one subfault, the response of the rest of fault can be saved, so we can speed the calculation of objective function by nearly 100 times. Second, as Sen and Stoffa (1995) pointed out, it is good for problems with a large number of free parameters. This method permits us to simultaneously and efficiently invert for the slip amplitude, slip direction, rise-time, and rupture velocity.

\section{Resolution Analysis}

In this section, we check the resolution and robustness of our new procedure by inverting synthetic data sets for two different source geometries. In the first test, we stress the reliability of the inversion method by returning to the fault geometry of Northridge earthquake discussed earlier. In the second test, we emphasize the effect of uncertainty caused by choice of local velocity structure on synthetic waveform and GPS measurement, assuming the geometry appropriate for the 1999 Hector Mine earthquake dataset.

\section{Strong Motion Inversion: 1994 Northridge Earthquake}

We use the station distribution and fault geometry of 1994 Northridge earthquake (Fig. 5). In this event, 30 strong-motion stations were within $40 \mathrm{~km}$ from the hypocenter, forming a good near-source station coverage. It should be pointed out that a good distribution of stations is always one of the fundamental conditions for a detailed analysis. The test rupture model is modified from finite-fault inversion analysis of Northridge earthquake by Wald and Heaton (1994) (Fig. 6). The fault plane is $18 \mathrm{~km}$ along strike and $24 \mathrm{~km}$ down dip, and the dip and strike of it are $40^{\circ}$ and $122^{\circ}$, respectively. We divide it into 196 subfaults with dimensions 1.29 by $1.71 \mathrm{~km}$, and each of these is represented by the four parameters already mentioned. In total, there are 784 free parameters.

We use the hard-rock velocity model (Wald et al., 1996) to generate the synthetic seismograms applying the FK code (Zhu and Rivera, 2001) with a 0.1-sec sample interval. In the inversion, we match the wavelet coefficients for the scale region from 0.8 to $12.8 \mathrm{sec}$ or in the period region from 0.04 to $1.2 \mathrm{~Hz}$. Because we only try to fit the 12.8 - to 25.6 -sec seismic signals, for each trace, we need to fit 31 to 63 wave- let coefficients. The total number of data points used in the inversion is about 4200.

During the test, bounds of 0 to $3 \mathrm{~m}$ are allowed for the slip amplitudes; the rise times vary from 0.5 to $3.5 \mathrm{sec}$ at a 0.5 -sec interval; the rake angle is given the range of $70^{\circ}$ to $140^{\circ}$; the rupture time of each subfault is bounded by the time for a rupture to reach the subfault from the hypocenter traveling at 2.4 to $3.6 \mathrm{~km} / \mathrm{sec}$. The inversion begins with a random model in the above domains. We let the inversion stop if the improvement is less than $0.05 \%$ in 20 iterations. During the inversions, we let the $W_{\mathrm{c}}$ be zero, so no smoothing or minimum moment constraints are used.

We show the inversion results of noise-free strongmotion data in Figures $6 \mathrm{c}$ and $6 \mathrm{~d}$. It recovers nearly all details and validates the algorithm and approach we used. In the next inversion, when we add $5 \%$ Gaussian noise (the peak amplitude of noise is $5 \%$ of peak amplitude of synthetic data in the time domain), the results are smeared (Figs. 6 and 6f); however, we found that the four parameters are affected differently. Rise time and rupture time, fault characteristics that are related to frequency variation in seismograms, are relatively stable for noise; i.e., the rupture time contours are nearly wholly recovered and the variation within rise time is within one sample interval $(0.5 \mathrm{sec})$. Absolute slip amplitudes and rake directions are affected, however.

\section{Sensitivity to Velocity Structure}

In recent work, Wald and Graves (2001) and Graves and Wald (2001) studied the importance of accurate Green's functions for finite-fault source inversions. They found that inaccurate 3D Green's functions allow only partial recovery of the slip distribution even when the rupture velocity, rise time, and rake angle are fixed; however, a joint geodetic and seismic inversion allows for reasonable recovery of slip distribution. This analysis prompts us to discuss the effect of Green's function before performing a combined inversion of 1999 Hector Mine earthquake in our companion article ( $\mathrm{Ji}$ et al., 2002).

It was pointed out that the velocity structure around the Hector Mine earthquake is relatively simple (Hauksson, 2000). The simple layered models can model the wavefield reasonably well. The southern California standard model (SoCal: Dreger and Helmberger, 1993), which is used in the automated source inversion for events in this region (Zhu and Helmberger, 1996), seems to be a good choice. The Mojave model (Jones and Helmberger, 1998) was shown to work well in explaining the waveforms from the Landers aftershock sequence, with similar paths for the Hector Mine earthquake, and could be another candidate. The differences between these two models are apparent (Fig. 7), particularly in the top $2.5 \mathrm{~km}$, where the SoCal model is $30 \%$ faster than the Mojave model. We assume such differences are representative of the possible velocity model uncertainties and we choose one of them to do the source inversion while treating the other one as test data. 

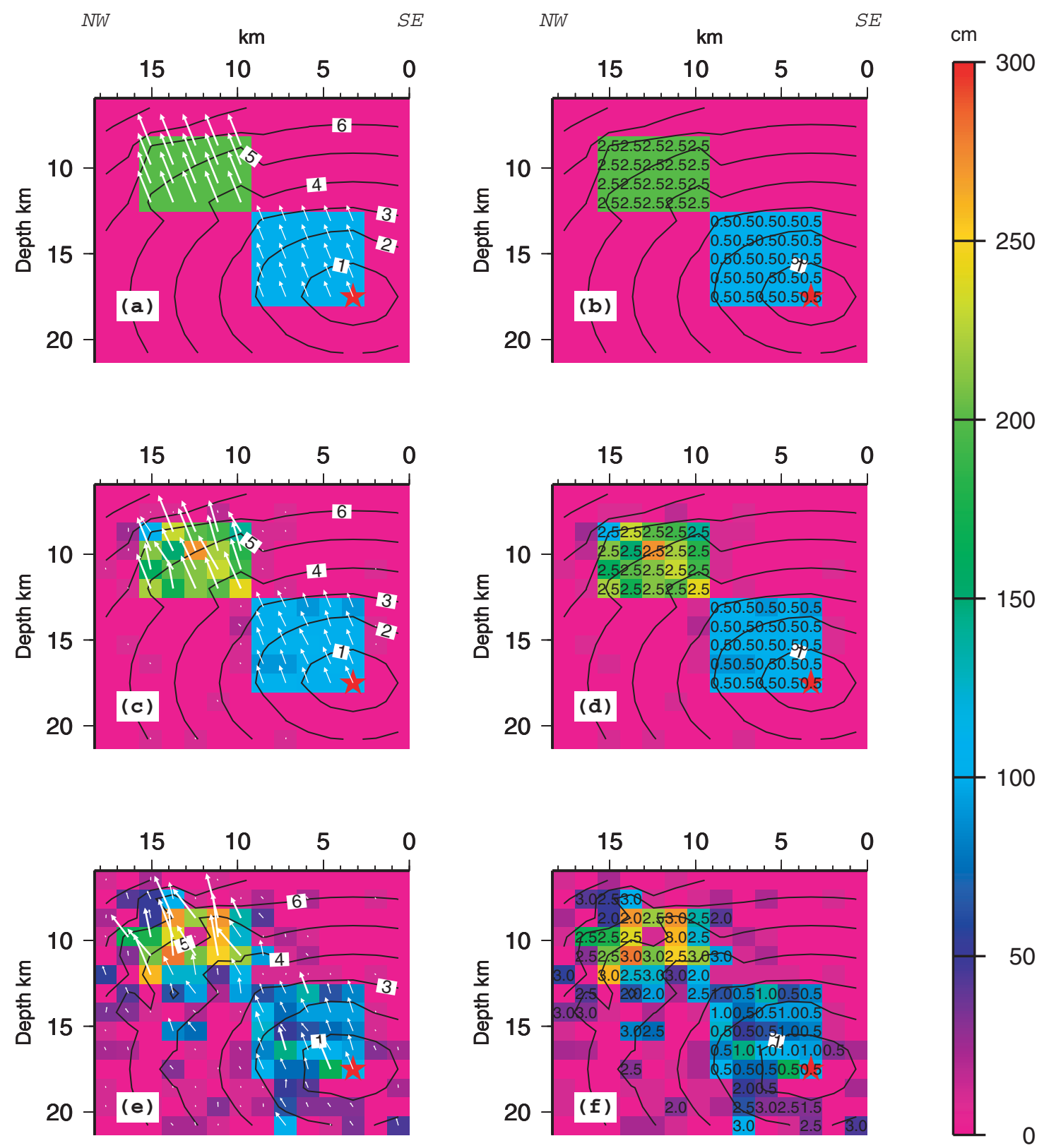

Figure 6. Comparison of finite fault inversions: (a) slip distribution and (b) rise time distribution of test model; (c) and (d) are results of noise free inversion; (e) and (f) are result of data contaminated with $5 \%$ Gaussian noise. The contours indicate the rupture initiations. The color shows the slip amplitudes, arrows indicate the slip directions. To highlight the major features, we add a $0.4-\mathrm{m}$ threshold before the plot; i.e., rise time is plotted only for subfault, with $>0.4 \mathrm{~m}$ slip. The average rupture velocities of subfaults with $<0.4 \mathrm{~m}$ slip is set to $2.6 \mathrm{~km} / \mathrm{sec}$, because it is poorly constrained when slips are small.

We use the same data distribution and similar fault geometry as used in the detailed analysis of the 1999 Hector Mine earthquake (Ji et al., 2002). The fault geometry shown in Figure 7 consists of three fault segments: fault 1 is in the central portion of fault system with a strike of $346^{\circ}$ and a dip of $85^{\circ}$; fault 2 is in the northwest, with a strike of $322^{\circ}$ and a dip of $75^{\circ}$; fault 3 is in the southeast, with a strike of $325^{\circ}$ and a dip of $85^{\circ}$. The total fault is subdivided into 168 elements, each with dimensions 3 by $2.7 \mathrm{~km}$. We use four parameters to determine the response of each subfault as mentioned above, so the total number of free parameters is 672 .

Within this fault geometry, we build a test model including four asperities; the slip on the rest of fault is set to zero. We fix the rake angles of all asperities to $175^{\circ}$, but each asperity has an individual rise time and slip amplitude. 

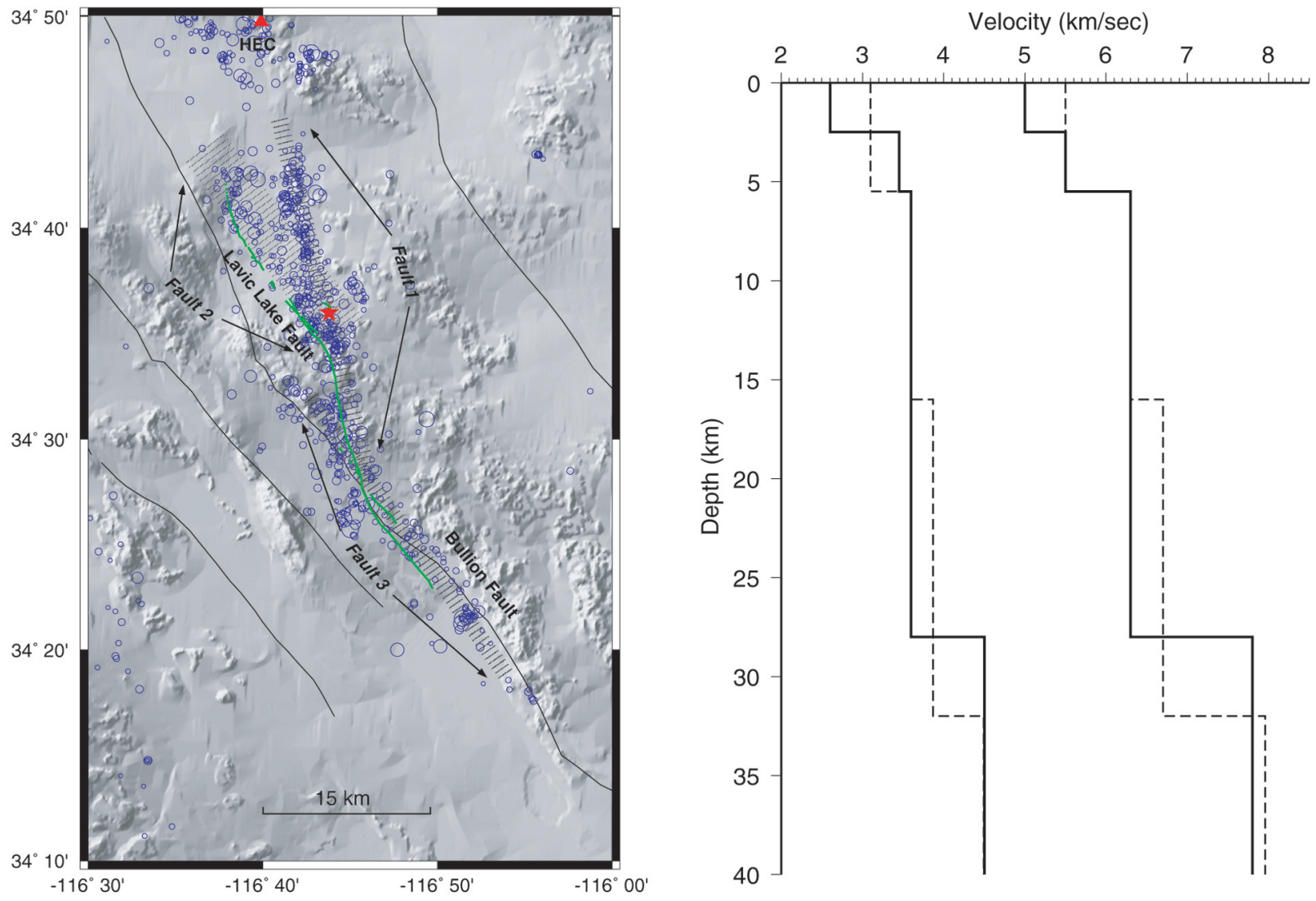

Figure 7. (Left) Test fault geometry for the Hector Mine earthquake on a base map of local topography. Thin lines show the major faults, the green trace indicates the mapped surface break, and a star is used to display the epicenter. The surface projections of the fault plane are presented with hachured lines. The blue circles show the aftershocks $\left(M_{\mathrm{L}}>2\right.$, Hauksson et al., 2002). The red triangle in the north indicates the location of TriNet station HEC. (Right) Velocity models. The solid lines show the Mojave model (Jones and Helmberger, 1998) and the dashed lines show the Southern California standard model (SoCal; Dreger and Helmberger, 1993).

Moreover, the rupture velocities are also varied asperity to asperity. For instance, rupture front speed is only $1.8 \mathrm{~km} /$ sec for the asperity on fault 2 . Finally, the total moment is $3.33 \times 10^{19} \mathrm{Nm}$ with the rigidity of the SoCal model, about one-half that of Hector Mine earthquake (Figs. 8a and 8b).

Records of 10 strong-motion and 38 GPS stations (Fig. 9) are used. The azimuth coverage of strong-motion stations is quite good, but we have only one station, HEC, whose epicentral distance is less than $30 \mathrm{~km}$. In addition, 15 teleseismic $P$ waves and 11 teleseismic SH waves are also used (Fig. 11).

We use the SoCal model to build the test data of the aforementioned fault model. The strong-motion, teleseismic $P$ or SH body-wave, and static displacements (Figs. 9, 10, and 11) of this layered model are generated with the synthetic methods developed by Zhu and Rivera (2001), Yao and Ji (1998), and Xie and Yao (1989). The Mojave model is then used to make the Green's functions for the later inversions, as we do in the companion article (Ji et al., 2002).

Before performing the inversion, we forward calculated the synthetic seismograms and static displacements with the test model (Fig. 8). As expected, the variations in teleseismic waveforms are small, and so are the static displacements. Using the Mojave model instead of the SoCal model causes about 5\% amplitude error in static displacements, even though the difference between a half-space and Mojave model can produce a $15 \%$ amplitude error. The effect is significant for the local strong motions, however, where the soft, near-surface layer generated larger surface waves. With inaccurate velocity structure, the test model is no longer the model that can best fit the data.

During the inversion, the dislocation amplitude is allowed to vary from 0 to $8 \mathrm{~m}$; the rake angle varies from $140^{\circ}$ to $210^{\circ}$; the average rupture velocity is selected to range from 1.6 to $3.0 \mathrm{~km} / \mathrm{sec}$ at an interval of $0.1 \mathrm{~km} / \mathrm{sec}$; and the rise time is allowed to range from 0.6 to $6 \mathrm{sec}$ at an 0.6-s interval. Finally, for the purpose of comparison, we use the same weights $W_{\mathrm{st}}$ and $W_{\mathrm{c}}$ as we used in the companion study (Ji et al., 2002), where $W_{\mathrm{st}}=1$ and $W_{\mathrm{c}}=0.1$.

The wavelet coefficients in a scale range of 1.6 to 25.6 
$N W$
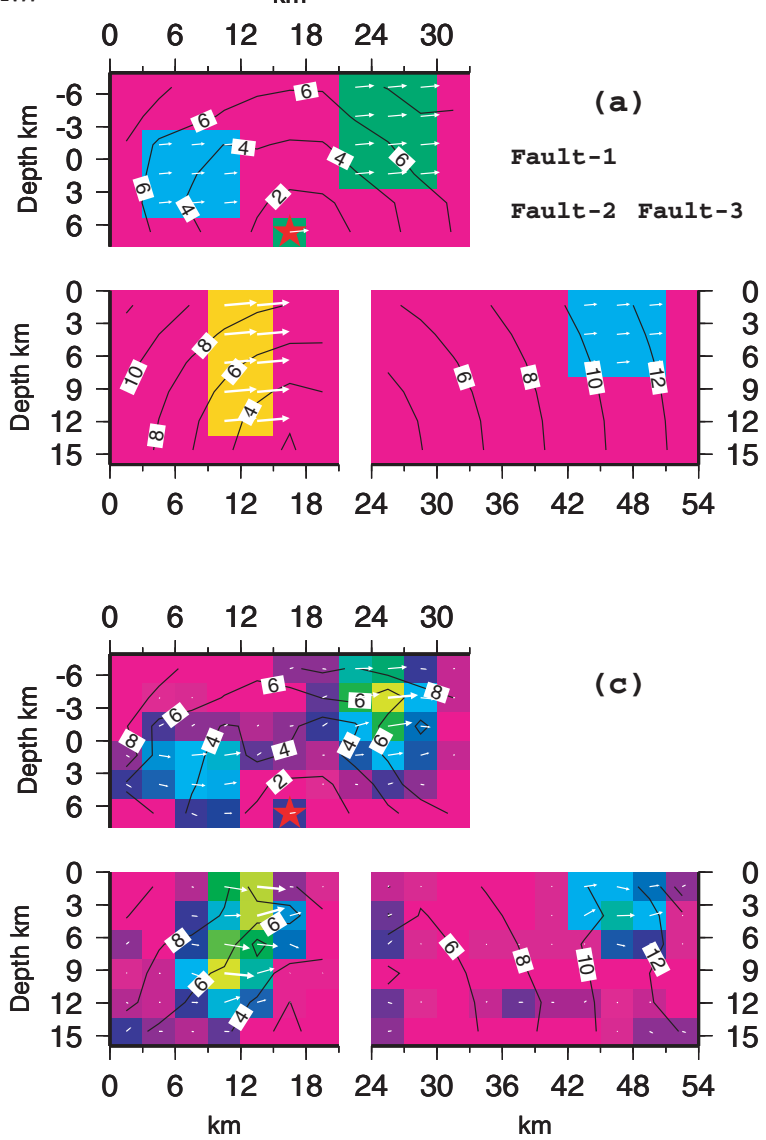

(a)

Fault-1

Fault-2 Fault-3

(c)

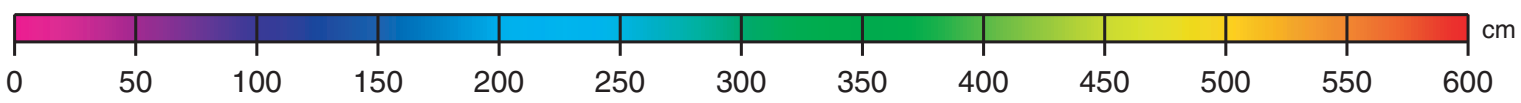

Figure 8. Cross section of the slip distribution of test models (top, a, b) and inverted models (bottom, c, d). Contours indicate the rupture time at a 2.0-sec interval. Slip amplitudes are displayed with color. At the left $(\mathrm{a}, \mathrm{c})$, the arrows are used to show the rake angles; and in the right $(b, d)$, the average rise time of each subfault is indicated. A $0.5-\mathrm{m}$ threshold is used before the plot; i.e., rise time is not plotted for subfaults with $<0.5 \mathrm{~m}$ slip, and the average rupture velocity of the fault segment is used to calculate the initiation time. sec for teleseismic $P$ waves and strong-motion data and 3.2 to $25.6 \mathrm{sec}$ for teleseismic SH waves are used to constrain the slip model. For the closest station, HEC, the coefficients of the wavelets with an 0.8 -sec scale are also used. The L1 + L2 combined criteria are used to constrain the wavelet coefficients with the scale greater than $3.2 \mathrm{sec}$, and the correlation function is used to constrain the fit to coefficients with scales of 0.8 and 1.6 sec. Finally, the GPS measurements offer 114 additional constraints to the slip distribution.

Three inversions with different initiation models are performed; all inverted models have smaller objective function values than that of the test model. One typical result is given in Figure 8. The synthetics generated from this model are displayed in Figure 9 by red arrows, Figure 10 by red lines, and Figure 11 by thin lines. Note that the inverted model matches the strong-motion data much better than the test model does (Fig. 10), but becomes worse at fitting the teleseismic data (Fig. 11). Finally, two models explain the GPS data equally well (Fig. 9).

Comparison between the test model and inverted model (Fig. 8) emphasizes the importance of a suitable velocity structure for source inversion studies. In a relative sense, the inversion for the slip distribution is more robust; the slip pattern is similar to the test model but is smeared. The recovery of the rise time and rupture velocity distributions is, however, strongly affected by the inaccurate velocity structure. Fortunately, the influence can be reduced if we have near-fault observations. For instance, in the four asperities, only that in the northern portion of fault 1 , which is near the closest strong-motion station, HEC, has a reasonable recovery of both rise time and rupture initiation contours. The resolution of the two asperities on fault 1 and 2 is also fairly 


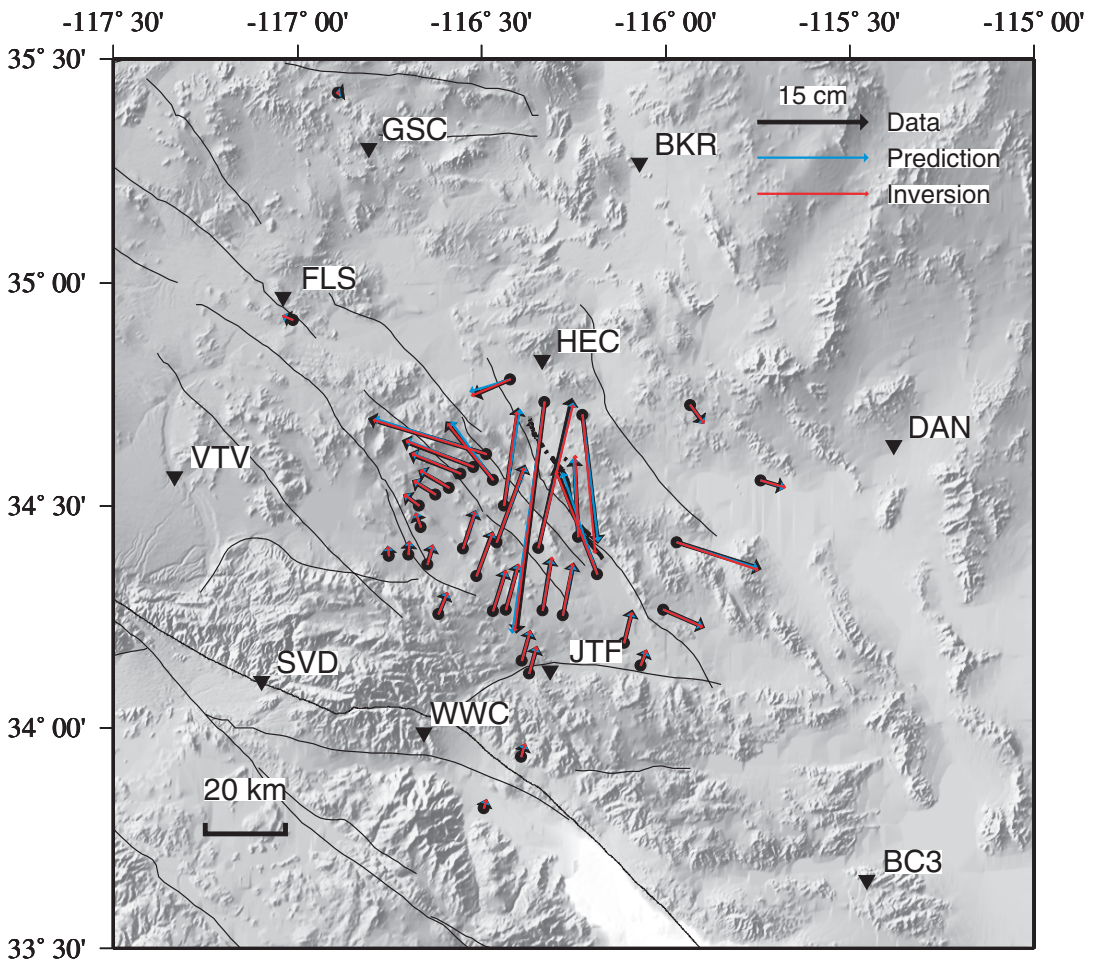

Figure 9. Distribution of strong-motion stations (triangles) and GPS stations (circles). The major faults in this regions are plotted by thin lines, and the surface break during Hector mine earthquake is indicated by a thick line. For each station, a black arrow shows synthetic displacement generated from the test model and SoCal velocity model; a blue arrow shows that from the test model and Mojave model; and a red arrow shows that from the inverted model and Mojave model. See text for details.

good, but significant variations are also observed. For a more quantitative evaluation, we calculate five basic parameters: seismic moment, average slip, average rake angle, rupture velocity, and rise time (Table 1). Because only the subfaults with larger dislocation amplitudes are well determined, the latter three parameters have been weighted with slip amplitudes:

$$
\begin{array}{r}
V_{\mathrm{m}}=\frac{\sum_{j} \sum_{k} D_{j k} V_{j k}}{\sum_{j} \sum_{k} D_{j k}}, \\
r_{\mathrm{m}}=\frac{\sum_{j} \sum_{k} D_{j k} r_{j k}}{\sum_{j} \sum_{k} D_{j k}}, \\
R_{\mathrm{m}}=\frac{\sum_{j} \sum_{k} D_{j k} R_{j k}}{\sum_{j} \sum_{k} D_{j k}} .
\end{array}
$$

Here, $D_{j k}, V_{j k}, r_{j k}$, and $R_{j k}$ are dislocation amplitude, rupture velocity, rise time, and rake angle of subfault $j k$, respectively.

The average retrieved values for the entire fault are well determined. About $10 \%$ difference in entire seismic moment release is caused by the different shear moduli of the models, because the average slip of inverted model is slightly larger than that of test model.

The effects of the inaccurate velocity model are appar- ent, but still reasonably small, when we consider the value of individual fault segments. The variations in rupture velocity are about $0.2 \mathrm{~km} / \mathrm{sec}$ (fault 2); the changes in rise time are less than about $0.4 \mathrm{sec}$ (fault 1). The differences in the average slip and rake angle are negligible for faults 1 and 2, but become larger for fault 3 , which is probably due to the fact that there is no strong-motion station near fault 3 .

With a large variation in velocity structure and few nearsouce strong-motion stations, the uncertainty of individual subfaults becomes quite large. Outside of the region of rupture initiation, it is difficult to have confidence in the characteristics of an individual subfault. However, if a group of subfaults have a similar characteristic, it is probably more

Table 1

Sensitivity of Finite Source Parameters to Variation in Velocity Structure

\begin{tabular}{lcccc}
\hline Parameter & Fault 1 & Fault 2 & Fault 3 & Whole Fault \\
\hline$M_{\mathrm{o}}\left(10^{26}\right.$ dyne cm) & $1.47(1.56)$ & $1.19(1.32)$ & $0.42(0.45)$ & $3.08(3.33)$ \\
Slip Amplitude $(\mathrm{cm})$ & $86(87)$ & $119(120)$ & $41(31)$ & $78(75)$ \\
Rake Angle $\left(^{\circ}\right)$ & $174(175)$ & $179(175)$ & $172(175)$ & $176(175)$ \\
Rupture Velocity & $2.3(2.3)$ & $2.0(1.8)$ & $2.7(2.8)$ & $2.23(2.17)$ \\
$\quad(\mathrm{km} / \mathrm{sec})$ & $2.3(2.7)$ & $2.5(2.4)$ & $2.9(3.0)$ & $2.50(2.60)$ \\
Rise time (sec) & & & & \\
\hline
\end{tabular}

The numbers in brackets are the corresponding input values of the test model. The moments are calculated based on velocity models that generate the synthetic seismograms and static displacements. The subfault dislocation amplitudes are used as weights to calculate the weighted average rake angle, rupture velocity, and rise time of whole fault and the three fault segments. 


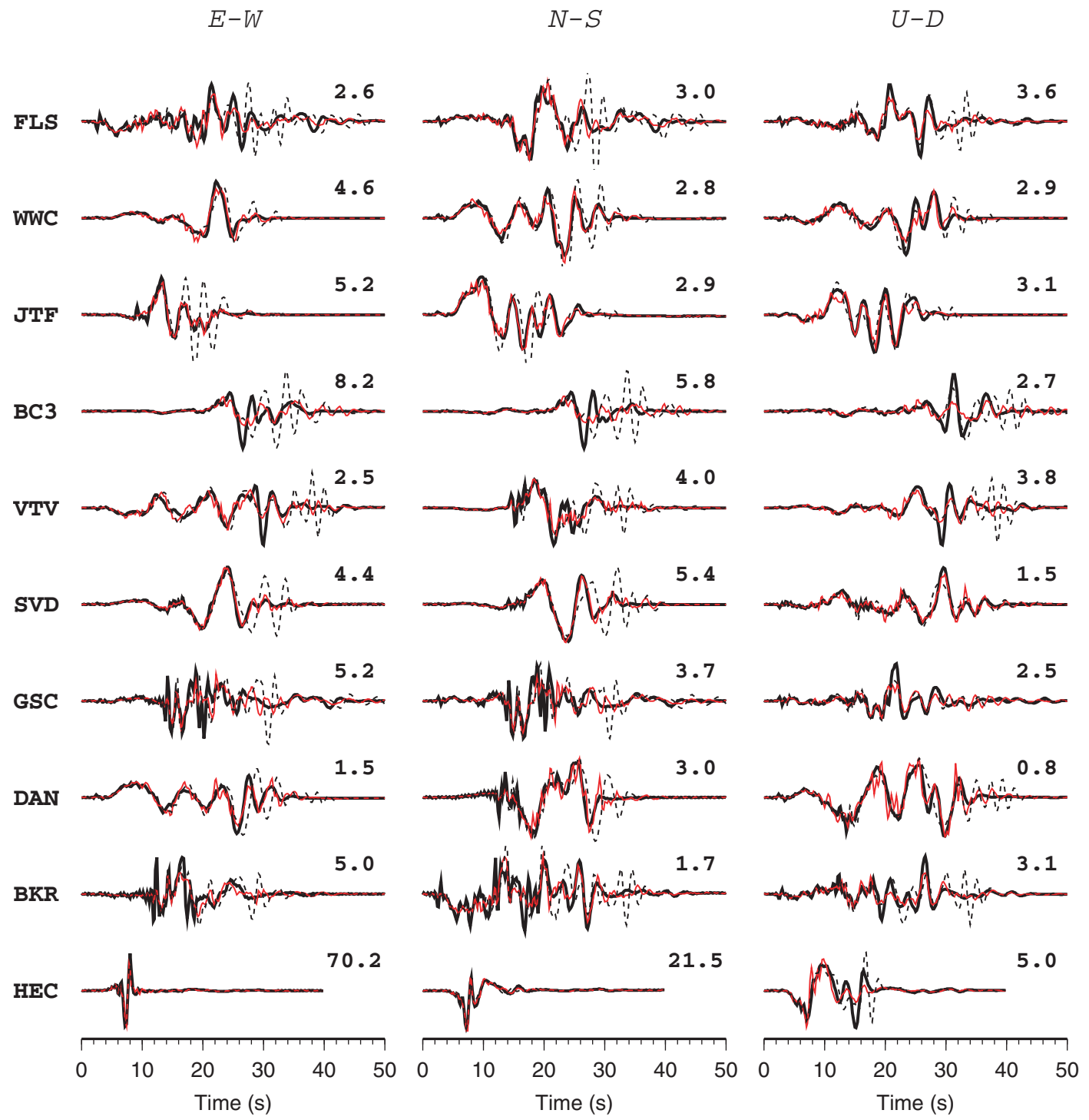

Figure 10. Strong motion velocity data (thick lines, generated by the test model and SoCal Model), forward prediction (dashed lines, generated by test model and Mojave model), and synthetics (red lines, generated by the inverted model and Mojave Model). The station names are indicated at the right of traces. The peak amplitudes of test data in $\mathrm{cm} / \mathrm{sec}$ are indicated above the end of traces.

\section{Discussion}

reliable. In addition, given an approximate velocity structure, we can recover the overall pattern fairly well.

In summary, from our resolution test, we can make several observations. First, except for the near-surface slip, which is strongly affected by the velocity structure, the recovery of the overall slip distribution is relatively robust. Second, rupture time and rise-time distributions are perturbed by the inaccurate velocity structure. Third, while individual subfault parameters may vary from the true model, overall features are still well-resolved. Finally, in this particular test, the variation of the average rupture velocity of one fault segment is less than $0.2 \mathrm{~km} / \mathrm{sec}$, and the variation in rise time is about $0.4 \mathrm{~s}$.
Inverting for source excitation with a limited knowledge of the Earth structure certainly proves challenging. While the primary faulting parameters are easily described, the details of the rupture process remain elusive. Incremental improvements in resolving for source complexity will be possible in the near future with the extensive deployments of modern geodetic and seismic networks combined with space-based observations, but additional advances in the inversion process will also be beneficial. Here, we have added to the source inversion tool chest by examining the variation of frequency content with time. In particular, the simultaneous inversion using the geodetic data to determine the slip distribution and the wavelet transform of the seismic data to 

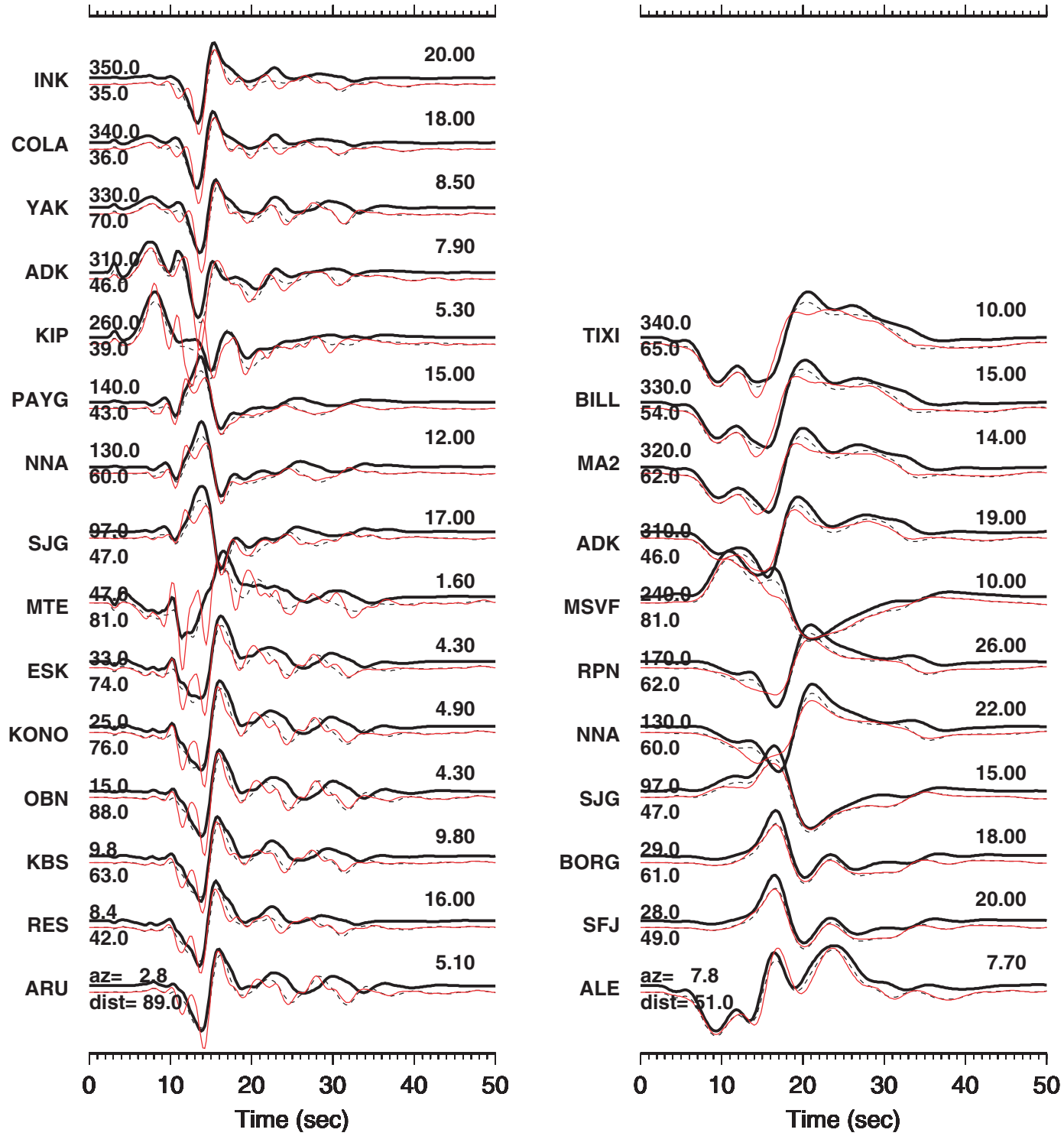

Figure 11. Teleseismic $P$ (left column) and SH (right column) velocity data (thick lines, generated by the test model and SoCal Model), forward prediction (dashed lines, generated by test model and Mojave model), and synthetics (red lines, generated by the inverted model and Mojave Model). The station abbreviations are indicated at the right of each trace. The peak amplitudes of test data in $\mathrm{mm} / \mathrm{sec}$ are indicated above the end of traces. The synthetic seismograms are slightly shifted in vertical for a better comparison.

address the temporal rupture properties prove beneficial. In our companion article (Ji et al., 2002), we apply this method to study the complex slip history of 1999 Hector Mine earthquake in detail.

It is noteworthy that the approach suggested in this work only begins to take advantage of the wavelet transform. Wavelet transform theory suggests that signals should be seen in a $2 \mathrm{D}$ time-frequency plane, instead of a 1D time or frequency axis. The criteria that measure the difference between observed and synthetic signals could reflect this concept. Compared with the approach used in this work, which weighted signals only by the signal scales, it could be done with both the scales and positions. For instance, the L2 norm in the wavelet domain could be

$$
e=\sum_{j} \sum_{k} W_{j, k}\left(o_{j, k}-s_{j, k}\right)^{2}
$$

Where $W_{j, k}$ is the weight related to scale parameter $j$ and position parameter $k$. With this approach, the prior knowledge of noise can be manipulated to zoom in on reliable signals and zoom out the large, noisy, contaminated signals 
by adjusting the weights. This approach will decrease the sensitivity to the velocity in some extent. Note that it is nearly impossible to accomplish this in the time or frequency domain alone.

Furthermore, since wavelet transform is a linear transform, it should be straightforward to apply this approach in other linear waveform inversion algorithms. Finally, the theory of wavelet transforms is still developing, and more wavelet atoms are being introduced. Since different wavelet atoms will focus on different qualities of seismic signals (e.g., time, frequency, or phase), introducing them into seismological studies will be helpful to better understanding the earthquake rupture process.

\section{Acknowledgments}

The project was supported by USGS and SCEC. Ji benefited from the discussion with Professor Zhenxing Yao (Institute of Geophysics, Chinese Academy of Sciences). The figures are made using GMT (Generic Mapping Tools) software (Wessel and Smith, 1991). This manuscript was prepared with AGU's $\mathrm{L}^{\mathrm{A}} \mathrm{T}_{\mathrm{E}} \mathrm{X}$ macros v5, with the extension package $\mathrm{AGU}^{++}$by P. W. Daly, version 1.6b, from 19 August 1999. This project was supported by SCEC contract No. NSF EAR-8920136 and by the U.S. Geological Survey under Contract No. 1HQGR0098. Contribution 8749, Division of Geological and Planetary Sciences, California Institute of Technology.

\section{References}

Aki, K., and P. G. Richards (1980). Quantitative Seismology: Theory and Methods, Vol. 2, W. H. Freeman and Co, New York, 813 pp.

Beroza, G. C. (1991). Near-source modeling of the Loma Prieta earthquake: evidence for heterogeneous slip and implications for earthquake hazard, Bull. Seism. Soc. Am. 81, 1603-1621.

Beroza, G. C., and P. Spudich (1988). Linearized inversion for fault rupture behavior: application to the 1984 Morgan Hill, California, earthquake, J. Geophys. Res. 93, 6275-6296.

Cohee, B. P., and G. C. Beroza (1994). Slip distribution of the 1992 Landers earthquake and its implications for earthquake source mechanics, Bull. Seism. Soc. Am. 84, 692-712.

Cotton, F., and M. Campillo (1995). Frequency domain inversion of strong motions: application to the 1992 Landers earthquake, J. Geophys. Res. 100(B3), 3961-3975.

Dreger, D. S., and D. V. Helmberger (1993). Determination of source parameters at regional distances with three-component sparse network data, J. Geophys. Res. 98, 8107-8125.

Graves, R. W., and D. J. Wald (2001). Resolution analysis of finite fault source inversion using 1D and 3D Green's functions. I. Strong motion, J. Geophys. Res. 106, 8745-8766.

Guatteri, M., and P. Spudich (2000). What can strong-motion data tell us about slip-weakening fault-friction law? Bull. Seism. Soc. Am. 90, 98116.

Hartzell, S. H., and T. H. Heaton (1983). Inversion of strong ground motion and teleseismic waveform data for the fault rupture history of the 1979 Imperial Valley, California earthquake, Bull. Seism. Soc Am. 73, $1553-1583$.

Hartzell, S. H., P. Liu, and C. Mendoza (1996). The 1994 Northridge, California, earthquake: investigation of rupture velocity, rise time, and high-frequency radiation, J. Geophys. Res. 101, no. B9, 20,09120,108 .

Hauksson, E. (2000). Crustal structure and seismicity distribution adjacent to the Pacific and North America plate boundary in southern California, J. Geophys. Res. 105, 13,875-13,903.

Hauksson, E., L. M. Jones, and K. Hutton (2002). The $1999 M_{\mathrm{w}} 7.1$ Hector
Mine, California, earthquake sequence: complex conjugate strike-slip faulting, Bull. Seism. Soc Am. 92, 1154-1170 (this issue).

Heaton, T. H. (1990). Evidence for and implications of self-healing pulses of slip in earthquake rupture, Phys. Earth Planet. Inter. 64, 1-20.

Hudnut, K. W., Y. Bock, M. Cline, P. Fang, J. Freymueller, K. Gross, D. Jackson, S. Larson, M. Lisowski, Z. Shen, and J. Svarc (1996) Coseismic displacements of 1994 Northridge, California earthquake, Geol. Soc. Am. Bull. 86, no. 1B, S19-S36.

Ji, C., D. J. Wald, and D. V. Helmberger (2002). Source description of the 1999 Hector Mine, California earthquake. II. Complexity of slip history, Bull. Seism. Soc. Am., 92, 1208-1226 (this issue).

Jones, L. E., and D. V. Helmberger (1998). Earthquake source parameters and fault kinematics in the eastern California shear zone, Bull. Seism. Soc. Am. 88, no. 8, 1337-1352.

Mallat, S. (1998). A Wavelet Tour of Signal Processing, Academic Press, San Diego.

Mendoza, C., and S. Hartzell (1988). Inversion for slip distribution using teleseismic $P$ waveforms: North Palm Springs, Borah Peak, and Michoacan earthquakes, Bull. Seism. Soc. Am. 78, 1092-1111.

Olson, A. H., and J. H. Anderson (1988). Implications of frequency-domain inversion of earthquake ground motions for resolving the space-time dependence of slip on an extended fault, Geophys. J. 94, 443-455.

Olson, A. H., and R. Apsel (1982) Finite fault and inversion theory with applications to the 1979 Imperial Valley earthquake, Bull. Seism. Soc. Am., 72, 1969-2001.

Rothman, D. H. (1986). Automatic estimation of large residual statics corrections, Geophysics 51, 337-346.

Sen, M. K., and P. L. Stoffa (1991). Nonlinear one-dimensional seismic waveform inversion using simulated annealing, Geophysics 56(10), 1624-1638.

Sen, M. K., and P. L. Stoffa (1995). Global Optimization Methods in Geophysical Inversion, Elsevier Science B. V., Amsterdam, The Netherlands, $94 \mathrm{pp}$.

Wald, D. J., and R. W. Graves (2001). Resolution analysis of finite fault source inversion using 1D and 3D Green's functions. II. Combining seismic and geodetic data, J. Geophys. Res. 106, 8767-8788.

Wald, D. J., and T. H. Heaton (1994). Spatial and temporal distribution of slip for the 1992 Landers, California, earthquake, Bull. Seism. Soc. Am. 84, 668-691.

Wald, D. J., D. V. Helmberger, and T. H. Heaton (1991). Rupture model of the 1989 Loma Prieta earthquake from the inversion of strongmotion and broadband teleseismic data, Bull. Seism. Soc. Am. 81, no. $5,1540-1572$.

Wald, D. J., T. H. Heaton, and K. W. Hudnut (1996). A dislocation model of the 1994 Northridge, California, earthquake determined from strong-motion, GPS, and leveling-line data, Bull. Seism. Soc. Am. 86, S49-S70.

Wessel, P., and W. H. F. Smith (1991). Free software helps map and display data, EOS Trans. Am. Geophys. Union. 72, 445-446.

Xie, X., and Z. Yao (1989). A generalized reflection-transmission coefficient matrix method to calculate static displacement field of a dislocation source in a stratified half-space, Chin. J. Geophys. 32, 191205.

Yamada, M., and K. Ohkitani (1991). Orthonormal wavelet analysis of turbulence, Fluid Dyn. Res. 8, 101-115.

Yao, Z., and C. Ji (1998). The inversion problem of finite fault study in time domain, Chin. J. Geophys. 40, 691-701.

Yomogida, K. (1994). Detection of anomalous seismic phases by the wavelet transform, Geophys. J. Int. 116, 119-130.

Zhao, L.-S., and D. V. Helmberger (1994). Source estimation from broadband regional seismograms, Bull. Seism. Soc. Am. 84, 91-104.

Zhu, L., and D. V. Helmberger (1996). Advancement in source estimation techniques using broadband regional seismograms, Bull. Seism. Soc. Am. 86, 1634-1641.

Zhu, L., and L. A. Rivera (2001). Computation of dynamic and static displacement from a point source in multi-layered media, Geophys. J. Int. 148, no. 3, 619-627. 
Seismological Laboratory

California Institute of Technology

Mail Code 252-21

1200 E. California Blvd.

Pasadena, California 91125

(C.J., D.V.H.)
U.S. Geological Survey

525 S. Wilson Ave.

Pasadena, California 91106

(D.J.W.)

Manuscript received 6 November 2000. 